Bull. Soc. math. France

134 (2), 2006, p. 241-252

\title{
KÄHLER MANIFOLDS WITH SPLIT TANGENT BUNDLE
}

\author{
by Marco Brunella, Jorge Vitório Pereira \\ \& FrédÉRIC TOUZET
}

\begin{abstract}
This paper is concerned with compact Kähler manifolds whose tangent bundle splits as a sum of subbundles. In particular, it is shown that if the tangent bundle is a sum of line bundles, then the manifold is uniformised by a product of curves. The methods are taken from the theory of foliations of (co)dimension 1 .

RÉsumÉ (Variétés kähleriennes à fibré tangent scindé). - On étudie dans cet article les variétés kählériennes compactes dont le fibré tangent se décompose en somme directe de sous-fibrés. En particulier, on montre que si le fibré tangent se décompose en somme directe de sous-fibrés en droites, alors la variété est uniformisée par un produit de courbes. Les méthodes sont issues de la théorie des feuilletages de (co)dimension 1.
\end{abstract}

\section{Introduction}

We study in this paper compact Kähler manifolds whose tangent bundle splits as a sum of two or more subbundles. The basic result that we prove is the following theorem.

Texte reçu le 21 septembre 2004, accepté le 28 octobre 2004.

Marco Brunella, IMB, 9 Avenue Savary, 21078 Dijon (France).

Jorge Vitório Pereira, IMPA, Est. D. Castorina 110, 22460-320 Rio de Janeiro (Brasil). Frédéric Touzet, IRMAR, Campus de Beaulieu, 35042 Rennes (France).

2000 Mathematics Subject Classification. - 32Q30, 37F75, 53C12.

Key words and phrases. — Kähler manifolds, holomorphic foliations, uniformisation, integrability. 
THEOREM 1.1. - Let $M$ be a compact connected Kähler manifold. Suppose that its tangent bundle $T M$ splits as $D \oplus L$, where $D \subset T M$ is a subbundle of codimension 1 and $L \subset T M$ is a subbundle of dimension 1 . Then:

(i) If $D$ is integrable then $\widetilde{M}$, the universal covering of $M$, splits as $\widetilde{N} \times E$, where $E$ is a connected simply connected curve (the unit disc $\mathbb{D}$, the affine line $\mathbb{C}$ or the projective line $\mathbb{P})$. This splitting of $\widetilde{M}$ is compatible with the splitting of $T M$, in the sense that $T \widetilde{N} \subset T \widetilde{M}$ is the pull-back of $D$ and $T E \subset T \widetilde{M}$ is the pull-back of $L$.

(ii) If $D$ is not integrable then $L$ is tangent to the fibres of a $\mathbb{P}$-bundle.

This result will be the main ingredient in the proof of the following one. See also Section 4 for a more general statement.

THEOREM 1.2. - Let $M$ be a compact connected Kähler manifold whose tangent bundle splits as a sum of line subbundles:

$$
T M=L_{1} \oplus \cdots \oplus L_{n} .
$$

Then the universal covering $\widetilde{M}$ is isomorphic to a product of curves

$$
\widetilde{M}=\mathbb{P}^{r} \times \mathbb{C}^{s} \times \mathbb{D}^{t}
$$

for suitable integers $r, s, t, r+s+t=n$. Moreover, if all the codimension 1 subbundles $L_{1} \oplus \cdots \oplus L_{j-1} \oplus L_{j+1} \oplus \cdots \oplus L_{n}, j=1, \ldots, n$, are integrable, then the above splitting of $\widetilde{M}$ is compatible with the one of $T M$.

The problem of relating splitting properties of the tangent bundle of a compact complex manifold with splitting properties of the universal covering has been recently studied by Beauville [2], Druel [9], Campana-Peternell [7]. The point of view of these papers consists in analysing the interplay between splitting of the tangent bundle and some known differential-geometric or algebraicgeometric properties of the manifold. For instance, in [2] one makes use of Kähler-Einstein metrics, whereas in [9] and [7] a main tool is the geometry of rational curves on a projective variety (Mori theory).

Our point of view is completely independent on the geometry of the underlying manifold. On the contrary, it is completely dependent on the geometry of the foliations by curves generated by one dimensional subbundles of the tangent bundle. In some sense, we replace the Mori theory used in [7] with the "foliated" Mori theory funded by Miyaoka [3]. But also we like to work on compact Kähler manifolds which are possibly nonprojective, so that the algebraic point of view of [3] must be replaced by the more analytic one of [5] and [6], which moreover gives some useful metric-type information. Other simple but essential tools are the integrability criterion for codimension 1 distributions of [8] and the construction of holonomy invariant metrics for codimension 1 foliations of $[4]$.

TOME $134-2006-\mathrm{N}^{\mathrm{O}} 2$ 
Roughly speaking, in the setting of Theorem 1.1 our method consists in constructing a special metric on the line bundle $L$. Then, in the setting of Theorem 1.2 and still roughly speaking, we shall obtain a special metric on $M$ by summing the special metrics on the line bundles $L_{j}$, and this special metric on $M$ will give the desired uniformisation. In this perspective, Theorem 1.2 should be compared with Simpson's uniformisation theorem [13, Cor. 9.7] (see also [2]), even if our construction of special metrics is completely different. In fact, we already have by free a special metric, given by the leafwise Poincaré metric, and we have just to verify that it is the good one.

\section{One dimensional foliations with a transverse distribution}

Let $M$ be a compact connected Kähler manifold. Suppose that the tangent bundle $T M$ splits as a sum of a one dimensional subbundle $L$ and a codimension 1 subbundle $D$ :

$$
T M=D \oplus L .
$$

The line subbundle $L$ is tangent to a holomorphic one dimensional foliation $\mathcal{L}$. Each leaf of $\mathcal{L}$ is uniformized either by $\mathbb{P}$ (rational leaf) or by $\mathbb{C}$ (parabolic leaf) or by $\mathbb{D}$ (hyperbolic leaf). By a well known argument (Reeb stability plus compactness of the cycles space [11]), if some leaf is rational then every leaf is rational, and $\mathcal{L}$ is a locally trivial $\mathbb{P}$-bundle over some compact connected Kähler manifold $N, \operatorname{dim} N=\operatorname{dim} M-1$.

In this case, the transverse distribution $D$ may be integrable or not. If it is integrable, then foliation $\mathcal{D}$ generated by $D$ can be described as a suspension of a representation of $\pi_{1}(N)$ into $\operatorname{Aut}(\mathbb{P})$, see $[10, \mathrm{Ch}$. I]. It follows that $\widetilde{M}$, the universal covering of $M$, splits as $\widetilde{N} \times \mathbb{P}$, the splitting being compatible with the splitting of $T M$.

If $D$ is not integrable and $M$ is projective, a more subtle argument $[7, \S 2]$ shows that $\widetilde{M}$ still splits as $\widetilde{N} \times \mathbb{P}$ (but now, of course, this splitting is no more compatible with $T M=D \oplus L)$. Probably the same holds also in the Kähler nonprojective case, but we don't know a proof.

Let us now turn to the more interesting case in which no leaf is rational. We shall distinguish two different possibilities:

(a) There is a hyperbolic leaf;

(b) Every leaf is parabolic.

The following Proposition completes the proof of Theorem 1.1.

Proposition 2.1. - In both cases (a) and (b) the distribution D is integrable, and generates a codimension 1 foliation $\mathcal{D}$. The holonomy of this foliation preserves a transverse hermitian metric of constant curvature $\kappa$, with $\kappa=-1$ in case (a) and $\kappa=0$ in case (b). The universal covering $\widetilde{M}$ splits as $\widetilde{N} \times E$, compatibly with $T M=D \oplus L$, and $E=\mathbb{D}$ in case (a) or $E=\mathbb{C}$ in case (b). 
2.1. The hyperbolic case. - If some leaf of $\mathcal{L}$ is hyperbolic, we shall rely on the main result of [5]: the leafwise Poincaré metric on $\mathcal{L}$ induces on $T^{*} \mathcal{L}$ $\left(=L^{*}\right)$ a singular hermitian metric whose curvature is a closed positive current.

Let us fix an open covering $\left\{U_{j}\right\}$ of $M$, with holomorphic vector fields $v_{j} \in H^{0}\left(U_{j}, \Theta_{M}\right)$ generating $\mathcal{L}$ and holomorphic 1-forms (a priori, not necessarily integrable) $\omega_{j} \in H^{0}\left(U_{j}, \Omega_{M}^{1}\right)$ generating $D$. We may suppose, by the transversality condition, that $i_{v_{j}} \omega_{j} \equiv 1$. On overlapping charts we therefore have

$$
v_{i}=g_{i j} v_{j}, \quad \omega_{i}=g_{i j}^{-1} \omega_{j}
$$

where $g_{i j}: U_{i} \cap U_{j} \rightarrow \mathbb{C}^{*}$ are holomorphic functions forming a cocycle which defines the line bundle $L^{*}$.

For every $j$, set

$$
h_{j}=\log \left\|v_{j}\right\|_{\text {Poin }}^{2}
$$

where $\left\|v_{j}(z)\right\|_{\text {Poin }}$ is the norm of $v_{j}(z)$ with respect to the Poincaré metric on the leaf of $\mathcal{L}$ through $z$. The result of [5] recalled above says that $h_{j}$ is a plurisubharmonic function. Recall also that, by definition, the Poincaré "metric" on a parabolic leaf is identically zero. Thus $h_{j}$ may have poles, corresponding to the trace of parabolic leaves on $U_{j}$.

The arguments are very close to [4] and [8]. In fact, the integrability of $D$ follows from [8] ( $L^{*}$ is the conormal bundle of $D$, and it is pseudoeffective), and the existence of a transverse metric invariant by the holonomy follows from [4, $\S \S 6-7]$. But let us give anyway some detail for the sake of completeness and reader's convenience.

From $v_{i}=g_{i j} v_{j}$ we deduce that $h_{i}-h_{j}=\log \left|g_{i j}\right|^{2}$, and from $\omega_{i}=g_{i j}^{-1} \omega_{j}$ we see that the $(1,1)$-form locally defined by

$$
\eta=\sqrt{-1} \mathrm{e}^{h_{j}} \omega_{j} \wedge \bar{\omega}_{j}
$$

is indeed a well defined global positive $(1,1)$-form (with $L_{\text {loc }}^{\infty}$-coefficients) on $M$. We may compute $\sqrt{-1} \partial \bar{\partial} \eta$, as a current. It turns out that it is a positive current.

Indeed, by the usual decomposition properties of positive forms, by $\eta \in L_{\text {loc }}^{\infty}$, and by Fubini's theorem, it is sufficient to verify that for every local embedding $\iota: \mathbb{D}^{2} \rightarrow M$ the current $\sqrt{-1} \partial \bar{\partial}\left(\iota^{*} \eta\right)$ is positive (that is, a positive measure on $\left.\mathbb{D}^{2}\right)$. If $\iota\left(\mathbb{D}^{2}\right)$ is tangent to $D$ then $\iota^{*} \eta \equiv 0$. If $\iota\left(\mathbb{D}^{2}\right)$ is not tangent to $D$ then the trace of $D$ on $\iota\left(\mathbb{D}^{2}\right)$ defines a foliation outside a discrete subset $\Gamma \subset \mathbb{D}^{2}$. Thus, $\iota^{*} \omega_{j}$ outside $\Gamma$ can be written, in suitable local coordinates $(z, w)$, as $f \mathrm{~d} z$, for some holomorphic function $f$. Consequently, $\iota^{*} \eta=\mathrm{e}^{h}|f|^{2} \sqrt{-1} \mathrm{~d} z \wedge \mathrm{d} \bar{z}$ and

$$
\sqrt{-1} \partial \bar{\partial}\left(\iota^{*} \eta\right)=\sqrt{-1} \partial \bar{\partial}\left(\mathrm{e}^{h+\log |f|^{2}}\right) \wedge \sqrt{-1} \mathrm{~d} z \wedge \mathrm{d} \bar{z}
$$

which is positive because $h+\log |f|^{2}$ is plurisubharmonic.

This gives the positivity of $\sqrt{-1} \partial \bar{\partial}\left(\iota^{*} \eta\right)$ on $\mathbb{D}^{2} \backslash \Gamma$. To obtain the positivity on the whole $\mathbb{D}^{2}$ we may simply use the extension theorem of [1]. The form $\iota^{*} \eta$

TOME $134-2006-\mathrm{N}^{\mathrm{O}} 2$ 
has bounded coefficients, so that if $\Theta$ is a Kähler form on $\mathbb{D}^{2}$ then $\iota^{*} \eta-c \Theta$ is a negative current for $c \gg 0$, whereas $\sqrt{-1} \partial \bar{\partial}\left(\iota^{*} \eta-c \Theta\right)=\sqrt{-1} \partial \bar{\partial}\left(\iota^{*} \eta\right)$ is positive outside $\Gamma$. By [1], this last one is positive on the full $\mathbb{D}^{2}$. Whence the positivity of $\sqrt{-1} \partial \bar{\partial} \eta$ on $M$.

By Stokes Theorem, the exact positive measure $\sqrt{-1} \partial \bar{\partial} \eta \wedge \Theta^{n-2}(\Theta$ is now a Kähler form on $M$, and $n=\operatorname{dim} M)$ must be identically zero, so that $\sqrt{-1} \partial \bar{\partial} \eta$ is also identically zero:

$$
\sqrt{-1} \partial \bar{\partial} \eta \equiv 0
$$

Looking again at the local restriction $\iota^{*} \eta, \iota: \mathbb{D}^{2} \rightarrow M$, we obtain that the function $\mathrm{e}^{h+\log |f|^{2}}$ is harmonic in the $w$-variable. Because $h+\log |f|^{2}$ is $w$ subharmonic, the only possibility is that $h+\log |f|^{2}$ is $w$-constant: the exponential of a nonconstant subharmonic function is strictly subharmonic. This implies that $\iota^{*} \eta$ is not only $\partial \bar{\partial}$-closed, but also $d$-closed. By varying the embedding $\iota: \mathbb{D}^{2} \rightarrow M$, we obtain:

$$
\mathrm{d} \eta \equiv 0
$$

This means two things:

(i) the distribution $D=\operatorname{ker} \eta$ is integrable, and hence generates a codimension 1 foliation $\mathcal{D}$;

(ii) on the transversals to $\mathcal{D}, \eta$ induces a measure invariant by the holonomy. Remark that all of this uses only the fact that $L^{*}$, the conormal bundle of $D$, is pseudoeffective, i.e., the functions $h_{j}$ are plurisubharmonic. But, by the normalisation $i_{v_{j}} \omega_{j} \equiv 1$ and the definition of $h_{j}$, we see that the restriction of $\eta$ to the leaves of $\mathcal{L}$ is nothing but than the area form of the hyperbolic metric on those leaves. Therefore, the holonomy of $\mathcal{D}$ preserves that hyperbolic metric.

In order to complete the proof of Proposition 2.1, case (a), it remains only to prove the splitting property of $\widetilde{M}$. This will follow from a general Splitting Lemma which we postpone to Section 3.

2.2. The parabolic case. - If all the leaves of $\mathcal{L}$ are parabolic, the leafwise Poincaré metric is identically zero and we cannot say, a priori, that $L^{*}$ is pseudoeffective (unless $M$ is projective, by [3]). But we shall see that indeed it is, and it is even flat, thanks to the existence of the transverse distribution $D$.

The starting point is the following one [6]: if $T \subset M$ is a codimension 1 disc transverse to $\mathcal{L}$, then the associated covering tube $U_{T}$ (union of the universal coverings of the leaves through $T$ ) is holomorphically trivial: $U_{T} \simeq T \times \mathbb{C}$. This fact can be reformulated in the following way. Take a foliated chart $U \simeq T \times \mathbb{D} \subset M$ around $T=T \times\{0\}$. Then any nonvanishing section $v_{0}$ of $\left.T \mathcal{L}\right|_{T}$ (i.e. a vector field tangent to $\mathcal{L}$ at points of $T$ ) can be extended to a section $v$ of $\left.T \mathcal{L}\right|_{U}$ in a canonical way: for every $t \in T$, we simply require that $\left.v\right|_{\{t\} \times \mathbb{D}}$ is the restriction to the plaque $\{t\} \times \mathbb{D}$ of a complete nonsingular vector field on the leaf of $\mathcal{L}$ containing $\{t\} \times \mathbb{D}$. This is well defined, because on a parabolic 
leaf the space of complete nonsingular vector fields is one dimensional. In other words, $v$ is obtained from $v_{0}$ by "parallel transport", using the natural affine structure on the leaves. Now, the trivialisation $U_{T} \simeq T \times \mathbb{C}$ means exactly that if $v_{0}$ is holomorphic then $v$ also is holomorphic.

We may cover $M$ with charts $U_{j}=T_{j} \times \mathbb{D}$ as above, and generate $\mathcal{L}$ with holomorphic vector fields $v_{j}$ as above. Thus $v_{i}=g_{i j} v_{j}$ on $U_{i} \cap U_{j}$. A moment of reflection shows that the cocycle $g_{i j}$ is constant along the (local) leaves of $\mathcal{L}$. In particular, $L^{*}$ is flat along the leaves of $\mathcal{L}$.

We also choose holomorphic 1-forms $\omega_{j} \in H^{0}\left(U_{j}, \Omega_{M}^{1}\right)$ generating $D$ and normalized by $i_{v_{j}} \omega_{j} \equiv 1$, so that $\omega_{i}=g_{i j}^{-1} \omega_{j}$. Hence

$$
\mathrm{d} \omega_{i}=-\frac{\mathrm{d} g_{i j}}{g_{i j}} \wedge \omega_{i}+g_{i j}^{-1} \mathrm{~d} \omega_{j}
$$

and by contracting with $v_{i}$, thanks to $\mathrm{d} g_{i j}\left(v_{i}\right) \equiv 0$, we find

$$
\frac{\mathrm{d} g_{i j}}{g_{i j}}=i_{v_{i}} \mathrm{~d} \omega_{i}-i_{v_{j}} \mathrm{~d} \omega_{j} .
$$

The global 2-form $\Omega$ locally defined by

$$
\Omega=\frac{1}{2 \pi \sqrt{-1}} \mathrm{~d}\left(i_{v_{j}} \mathrm{~d} \omega_{j}\right)
$$

represents, in De Rham's sense, the Chern class of $L^{*}, c_{1}\left(L^{*}\right) \in H^{2}(M, \mathbb{R})$. This class must belong, at the same time, to $H^{1,1}(M, \mathbb{C}$ ) (as any Chern class) and to $H^{2,0}(M, \mathbb{C})$ ( because $\Omega$ is holomorphic). Since $M$ is Kähler it follows from Hodge decomposition theorem that $H^{1,1}(M, \mathbb{R}) \cap H^{2,0}(M, \mathbb{C})=\{0\}$. Hence we deduce that $c_{1}\left(L^{*}\right)=0$, that is $L^{*}$ is flat.

Now we may proceed as in the hyperbolic case. By flatness, we have

$$
\log \left|g_{i j}\right|^{2}=h_{i}-h_{j}
$$

where $h_{j}$ are suitable pluriharmonic functions on $U_{j}$. Then the $(1,1)$-form

$$
\eta=\sqrt{-1} \mathrm{e}^{h_{j}} \omega_{j} \wedge \bar{\omega}_{j}
$$

is, as in the previous section, a closed $(1,1)$-form. It is worth noting that the computations here are much more simple, because $h_{j}=\log \left|H_{j}\right|^{2}$ for some holomorphic $H_{j}$, so that

$$
\eta=\sqrt{-1}\left(H_{j} \omega_{j}\right) \wedge \overline{\left(H_{j} \omega_{j}\right)} \quad \text { and } \quad \sqrt{-1} \partial \bar{\partial} \eta=\mathrm{d}\left(H_{j} \omega_{j}\right) \wedge \mathrm{d} \overline{\left(H_{j} \omega_{j}\right)} .
$$

The closedness of $\eta$ gives the integrability of $D$ and the transverse metric invariant by the holonomy. This transverse metric is flat because the functions $h_{j}$ are pluriharmonic.

Finally, the splitting property of $\widetilde{M}$ follows from the Splitting Lemma of Section 3 below.

TOME $134-2006-\mathrm{N}^{\mathrm{O}} 2$ 
REMARK 2.1. - We have used here only the fact that $L^{*}$ is defined by a cocycle locally constant on the leaves of $\mathcal{L}$. This is equivalent to say that the leaves of $\mathcal{L}$ have an affine structure depending holomorphically on the leaf. A priori, such a property is much weaker then the parabolicity of leaves: any noncompact curve possesses an affine structure, because it possesses a submersion to $\mathbb{C}$ ! But a posteriori, and using the transverse distribution $D$, we obtain that the leaves of $\mathcal{L}$ are necessarily parabolic because the flat metric transverse to $\mathcal{D}$ induces on each leaf of $\mathcal{L}$ a complete flat hermitian metric. We don't know, and would like to know, if such a property still holds without the transverse distribution $D$. That is: given a foliation $\mathcal{L}$ on a compact Kähler manifold, such that its leaves have an affine structure depending holomorphically on the leaf, is it true that these leaves are parabolic? Note that there are counterexamples in the non-Kähler case, e.g. foliations on Inoue surfaces [4]. The problem is that an affine structure is not necessarily unimodular, and so it does not necessarily correspond to a flat metric.

A dual problem is: given a codimension 1 foliation on a compact Kähler manifold, admitting a transverse affine structure invariant by the holonomy, is it true that this structure is unimodular? The conormal bundle of such a foliation is by definition flat, and the proof above shows that the answer is affirmative. The reader may find more material on these aspects in [12].

\section{The Splitting Lemma}

LEMMA 3.1. - Let $M$ be a compact connected complex manifold whose tangent bundle $T M$ splits as

$$
T M=D \oplus L_{1} \oplus \cdots \oplus L_{k}
$$

where $L_{1}, \ldots, L_{k}$ have dimension 1 and $D$ has codimension $k$. Suppose that:

(i) for every $j=1, \ldots, k$ the codimension 1 subbundle

$$
D^{(j)}=D \oplus L_{1} \oplus \cdots \oplus L_{j-1} \oplus L_{j+1} \oplus \cdots \oplus L_{k}
$$

is integrable, and generates a codimension 1 foliation $D^{(j)}$;

(ii) for every $j=1, \ldots, k$, the foliation $\mathcal{D}^{(j)}$ admits a transverse hermitian metric of constant curvature invariant by the holonomy.

Then the universal covering $\widetilde{M}$ of $M$ splits as

$$
\widetilde{M}=\widetilde{N} \times E_{1} \times \cdots \times E_{k}
$$

where each $E_{j}$ is either $\mathbb{D}$ or $\mathbb{C}$ or $\mathbb{P}$, and where $T \widetilde{N} \subset T \widetilde{M}$ is the pull-back of $D \subset T M$. Moreover, if $L_{1} \oplus \cdots \oplus L_{k}$ is integrable then $T E_{j} \subset T \widetilde{M}$ is the pull-back of $L_{j} \subset T M$, for every $j$.

Proof. - The integrability of all $D^{(j)}$ gives the one of $D=\cap_{j=1}^{k} D^{(j)}$, so that $D$ is tangent to a codimension $k$ foliation $\mathcal{D}\left(=\cap_{j=1}^{k} \mathcal{D}^{(j)}\right)$. Similarly, $D \oplus L_{j}$ is 
integrable for every $j$, so that the holonomy of $\mathcal{D}$ preserves the splitting of its normal bundle induced by $L_{1} \oplus \cdots \oplus L_{k}=T M / D$. By hypothesis (ii), we may put on the leaves of $\mathcal{L}_{j}$ (the foliation generated by $L_{j}$ ) an hermitian metric $g_{j}$ of constant curvature $\kappa_{j} \in\{-1,0,1\}$, preserved by the holonomy of $\mathcal{D}^{(j)}$. Then $g=\bigoplus_{j=1}^{k} g_{j}$ is a homogeneous hermitian metric on $T M / D$ preserved by $\mathcal{D}$.

The general theory of transversely homogeneous foliations [10, Ch. III] gives a submersion

$$
\pi: \widetilde{M} \rightarrow E_{1} \times \cdots \times E_{k}
$$

whose fibres are the leaves of $\widetilde{\mathcal{D}}$, with $E_{j}=\mathbb{D}\left(\right.$ if $\left.\kappa_{j}=-1\right), \mathbb{C}\left(\right.$ if $\left.\kappa_{j}=0\right)$ or $\mathbb{P}$ (if $\kappa_{j}=1$ ). The distribution $\widetilde{L}_{1} \oplus \cdots \oplus \widetilde{L}_{k} \subset T \widetilde{M}$ is transverse to the fibres of $\pi$, and each summand $\widetilde{L}_{j}$ projects isometrically to $T E_{j}$, with the appropriate constant curvature metric.

Consider the restriction of $\pi$ over some curve:

$$
\begin{gathered}
\mathbf{c}=\left(c_{1}, \ldots, c_{j-1}, c_{j+1}, \ldots, c_{k}\right) \in E_{1} \times \cdots \times E_{j-1} \times E_{j+1} \times \cdots \times E_{k}, \\
E_{j}^{\mathbf{c}}=\left\{\left(z_{1}, \ldots, z_{k}\right) \in E_{1} \times \cdots \times E_{k} \mid z_{i}=c_{i}, i \neq j\right\}, \quad \widetilde{M}_{j}^{\mathbf{c}}=\pi^{-1}\left(E_{j}^{\mathbf{c}}\right), \\
\pi_{j}^{\mathbf{c}}: \widetilde{M}_{j}^{\mathbf{c}} \longrightarrow E_{j}^{\mathbf{c}} .
\end{gathered}
$$

The foliation $\widetilde{\mathcal{L}}_{j}$ is tangent to $\widetilde{M}_{j}^{\mathbf{c}}$, and there it is transverse to the fibres of $\pi_{j}^{\mathbf{c}}$. On each leaf of $\widetilde{\mathcal{L}}_{j}$ the projection $\pi_{j}^{\mathbf{c}}$ is a local isometry to $E_{j}^{\mathbf{c}}$. Now, the basic fact is that the metric on the leaves of $\widetilde{\mathcal{L}}_{j}$, arising from the metric on the leaves of $\mathcal{L}_{j}$, is complete, by compactness of $M$. It follows that on each leaf of $\widetilde{\mathcal{L}}_{j}$, $\pi_{j}^{\mathbf{c}}$ is a covering map, hence a global isometry of the leaf with $E_{j}^{\mathbf{c}}$. Therefore the foliation $\widetilde{\mathcal{L}}_{j}$ induces a trivialisation

$$
\widetilde{M_{j}^{\mathbf{c}}} \simeq \widetilde{N} \times E_{j}^{\mathbf{c}}
$$

where $\tilde{N}$ is a fibre of $\pi_{j}^{\mathbf{c}}$ (all the fibres are now isomorphic). This splitting is, by construction, compatible with $T \widetilde{M}_{j}^{\mathrm{c}}=\widetilde{D} \oplus \widetilde{L}_{j}$.

By the same argument, if $L_{1} \oplus \cdots \oplus L_{k}$ is integrable we obtain

$$
\widetilde{M}=\widetilde{N} \times E_{1} \times \cdots \times E_{k}
$$

in a way compatible with $T \widetilde{M}=\widetilde{D} \oplus \widetilde{L}_{1} \oplus \cdots \oplus \widetilde{L}_{k}$. But we claim that such a splitting (without compatibility) exists even in the nonintegrable case, by an "iterated integral" argument.

Indeed, fix a fibre $\widetilde{N}$ of $\pi$, over some point $\left(c_{1}, \ldots, c_{k}\right) \in E_{1} \times \cdots \times E_{k}$. We already know that $\widetilde{M}_{1}^{\mathbf{c}}, \mathbf{c}=\left(c_{2}, \ldots, c_{k}\right)$, is a product, in a canonical way induced by $\widetilde{\mathcal{L}}_{1}$. Thus we have a canonical family of isomorphisms

$$
\varphi^{a_{1}}: \pi^{-1}\left(a_{1}, c_{2}, \ldots, c_{k}\right) \longrightarrow \tilde{N}, \quad a_{1} \in E_{1} .
$$

TOME $134-2006-\mathrm{N}^{\mathrm{O}} 2$ 
For every $a_{1} \in E_{1}$ we also know that $\widetilde{M_{2}^{\mathbf{c}^{\prime}}}, \mathbf{c}^{\prime}=\left(a_{1}, c_{3}, \ldots, c_{k}\right)$, has a product structure induced by $\widetilde{\mathcal{L}}_{2}$. Thus we have, for every $a_{1}$, a canonical family of isomorphisms

$$
\varphi_{a_{1}}^{a_{2}}: \pi^{-1}\left(a_{1}, a_{2}, c_{3}, \ldots, c_{k}\right) \longrightarrow \pi^{-1}\left(a_{1}, c_{2}, \ldots, c_{k}\right), \quad a_{2} \in E_{2}
$$

and consequently

$$
\varphi^{a_{1}, a_{2}}=\varphi^{a_{1}} \circ \varphi_{a_{1}}^{a_{2}}: \pi^{-1}\left(a_{1}, a_{2}, c_{3}, \ldots, c_{k}\right) \longrightarrow \tilde{N} .
$$

By iterating this process, we find for every $\left(a_{1}, \ldots, a_{k}\right)$ an isomorphism

$$
\varphi^{a_{1}, \ldots, a_{k}}: \pi^{-1}\left(a_{1}, \ldots, a_{k}\right) \longrightarrow \tilde{N}
$$

canonically defined by the foliations. This isomorphisms depend holomorphically on $\left(a_{1}, \ldots, a_{k}\right)$ (this is just the holomorphic dependence on the initial condition of the solution of a holomorphic ODE), and they provide the desired product structure on $\widetilde{M}$.

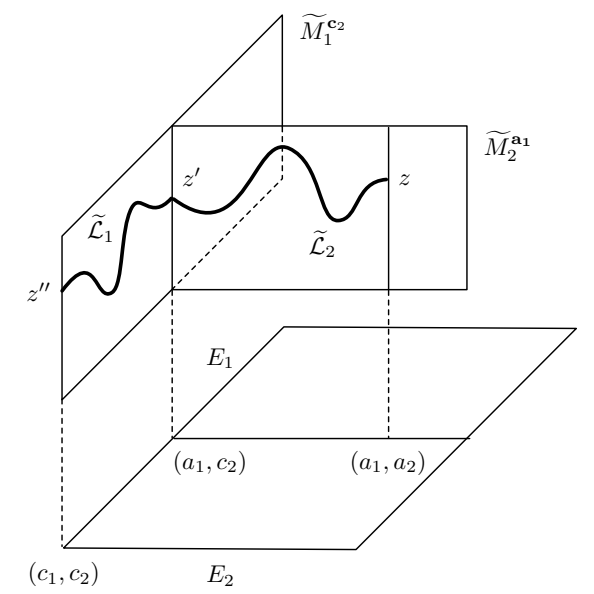

FiguRE 1. $z^{\prime}=\varphi_{a_{1}}^{a_{2}}(z), \quad z^{\prime \prime}=\varphi^{a_{1}}\left(z^{\prime}\right)=\varphi^{a_{1}, a_{2}}(z)$

There are evident generalisations of this lemma. We have done just the minimal amount of work for our purposes.

\section{Total Splitting}

Theorem 1.2 is a special case (with $D=\{0\}$ ) of the following more general result.

BULletin DE LA SOCiÉtÉ MATHÉmATiQUe DE FRANCE 
TheOREM 4.1. - Let $M$ be a compact connected Kähler manifold whose tangent bundle $T M$ splits as

$$
T M=D \oplus L_{1} \oplus \cdots \oplus L_{k}
$$

where $D \subset T M$ is a subbundle of codimension $k$ and $L_{j} \subset T M, j=1, \ldots, k$, are subbundles of dimension 1 . Then the universal covering $\widetilde{M}$ admits the structure of a $\left(\mathbb{P}^{r} \times \mathbb{C}^{s} \times \mathbb{D}^{t}\right)$-bundle over $R$, for a suitable complex manifold $R$ with $\operatorname{dim} R=\operatorname{dim} D$, and suitable integers $r, s, t, r+s+t=k$. Moreover, if all the codimension 1 subbundles $D \oplus L_{1} \oplus \cdots \oplus L_{j-1} \oplus L_{j+1} \oplus \cdots \oplus L_{k}, j=1, \ldots, k$, are integrable, as well as $L_{1} \oplus \cdots \oplus L_{k}$, then $\widetilde{M}$ splits as $R \times \mathbb{P}^{r} \times \mathbb{C}^{s} \times \mathbb{D}^{t}$, and this splitting is compatible with the one of $T M$.

Proof. - Suppose that one of the foliations $\mathcal{L}_{j}$ generated by $L_{j}$, say $\mathcal{L}_{k}$, is a foliation by rational curves, i.e. a $\mathbb{P}$-bundle over some compact connected Kähler manifold $M^{\prime}$, $\operatorname{dim} M^{\prime}=\operatorname{dim} M-1$. We claim that $T M^{\prime}$ splits as $D^{\prime} \oplus L_{1}^{\prime} \oplus \cdots \oplus L_{k-1}^{\prime}$.

To see this, recall that any vector bundle $V$ on $\mathbb{P}$ splits as $\mathcal{O}\left(m_{1}\right) \oplus \cdots \oplus$ $\mathcal{O}\left(m_{r}\right)$, where the multidegree $\left(m_{1}, \ldots, m_{r}\right) \in \mathbb{Z}^{r}$ is uniquely defined up to a permutation. It follows that if $V=V_{1} \oplus V_{2}$ then, necessarily, the multidegrees of $V_{1}$ and $V_{2}$ correspond to a decomposition in two disjoint pieces of the multidegree of $V$. Take now a fibre $F \simeq \mathbb{P}$ of $\pi^{\prime}: M \rightarrow M^{\prime}$, and consider $\left.T M\right|_{F}$, which splits as $\mathcal{O}(2) \oplus \mathcal{O}(0) \oplus \cdots \oplus \mathcal{O}(0)$, with $\mathcal{O}(2)=T F$. It also splits as $\left.\left.\left.T F \oplus D\right|_{F} \oplus L_{1}\right|_{F} \oplus \cdots \oplus L_{k-1}\right|_{F}$, and by the previous remark the multidegree of $\left.D\right|_{F}$ and the degrees of $\left.L_{j}\right|_{F}, j=1, \ldots, k-1$, are all equal to 0 . This means that they can be projected to $T_{\pi(F)} M^{\prime}$, giving the desired decomposition of $T M^{\prime}$.

Suppose that another one of the foliations $\mathcal{L}_{j}$, say $\mathcal{L}_{k-1}$, is a foliation by rational curves. Then the foliation $\mathcal{L}_{k-1}^{\prime}$ generated by $L_{k-1}^{\prime}$ is also a foliation by rational curves, and we have a $\mathbb{P}$-fibration $\pi^{\prime \prime}: M^{\prime} \rightarrow M^{\prime \prime}$. Consider the composition $\pi^{\prime \prime} \circ \pi^{\prime}: M \rightarrow M^{\prime \prime}$. Its fibres are $\mathbb{P}$-bundles over $\mathbb{P}$, but they are also leaves of the integrable distribution of $L_{k-1} \oplus L_{k}$ (integrability is just a restatement of projectability of $L_{k-1}$ along $L_{k}$ ). Hence, those fibres are isomorphic to $\mathbb{P} \times \mathbb{P}$. As before, the subbundles $D$ and $L_{j}, j=1, \ldots, k-2$, are projectable to $M^{\prime \prime}$.

By continuing in this way, we finally obtain, for some $r \geq 0$, a $\mathbb{P}^{r}$-bundle

$$
\pi: M \longrightarrow N
$$

such that:

(i) the fibres are tangent to those $L_{j}$ generating a foliation by rational curves, say $L_{k-r+1}, \ldots, L_{k}$

(ii) $D$ and $L_{j}, j=1, \ldots, k-r$, are projectable to $N$, giving a splitting

$$
T N=D^{\prime} \oplus L_{1}^{\prime} \oplus \cdots \oplus L_{k-r}^{\prime}
$$

TOME $134-2006-\mathrm{N}^{\mathrm{O}} 2$ 
(iii) no $L_{j}^{\prime}, j=1, \ldots, k-r$, generates a foliation by rational curves.

Set

$$
D^{(j)}=D^{\prime} \oplus L_{1}^{\prime} \oplus \cdots \oplus L_{j-1}^{\prime} \oplus L_{j+1}^{\prime} \oplus \cdots \oplus L_{k-r}^{\prime}
$$

so that $T N=D^{(j)} \oplus L_{j}^{\prime}$.

By Proposition 2.1, $D^{(j)}$ is integrable and generates a codimension 1 foliation whose holonomy preserves a transverse metric of curvature 0 or -1 . By the Splitting Lemma, the universal covering $\widetilde{N}$ splits as $R \times \mathbb{C}^{s} \times \mathbb{D}^{t}$, for a suitable $R$ and suitable integers $s, t$ with $s+t=k-r$.

Therefore, the universal covering $\widetilde{M}$ is a $\mathbb{P}^{r}$-bundle over $\widetilde{N}=R \times \mathbb{C}^{s} \times \mathbb{D}^{t}$, and consequently it is also a $\left(\mathbb{P}^{r} \times \mathbb{C}^{s} \times \mathbb{D}^{t}\right)$-bundle over $R$, because any $\mathbb{P}^{r}$-bundle over $\mathbb{C}^{s} \times \mathbb{D}^{t}$ is trivial.

The last sentence of the theorem is also easy to verify.

REMARK 4.1. - If $M$ is projective, then by $[7, \S 2] \widetilde{M}$ is a trivial bundle over $\widetilde{N}$, and therefore a trivial bundle over $R$.

\section{BIBLIOGRAPHY}

[1] Alessandrini (L.) \& Bassanelli (G.) - Plurisubharmonic currents and their extension across analytic subsets, Forum Math., t. 5 (1993), pp. $577-$ 602.

[2] Beauville (A.) - Complex manifolds with split tangent bundle, in Complex analysis and algebraic geometry, de Gruyter, Berlin, 2000, pp. 61-70.

[3] Bogomolov (F.) \& McQuillan (M.) - Rational curves on foliated varieties, Preprint IHÉS (2001).

[4] Brunella (M.) - Feuilletages holomorphes sur les surfaces complexes compactes, Ann. Sci. École Norm. Sup., t. 30 (1997), pp. 569-594.

[5] _ Plurisubharmonic variation of the leafwise Poincaré metric, Int. J. Math., t. 14 (2003), pp. 139-151.

[6] _ Some remarks on parabolic foliations, Contemp. Math., t. 389 (2005), pp. 91-102.

[7] Campana (F.) \& Peternell (T.) - Projective manifolds with splitting tangent bundle I, Math. Z., t. 241 (2002), pp. 613-637.

[8] Demailly (J.-P.) - On the frobenius integrability of certain holomorphic p-forms, in Complex geometry (Göttingen, 2000), Springer, Berlin, 2002, pp. 93-98.

[9] Druel (S.) - Variétés algébriques dont le fibré tangent est totalement décomposé, J. reine angew. Math., t. 522 (2000), pp. 9161-171.

[10] Godbillon (C.) - Feuilletages : études géométriques, in Progress in Mathematics, vol. 98, Birkhäuser Verlag, Basel, 1991. 
[11] Pereira (J. V.) - Global stability for holomorphic foliations on Kaehler manifolds, Qual. Theory Dyn. Syst., t. 2 (2001), pp. 381-384.

[12] - Fibrations, divisors and transcendental leaves, J. Algebraic Geom., t. 15 (2006), no. 1, pp. 87-110.

[13] Simpson (C.) - Constructing variations of Hodge structure using YangMills theory and applications to uniformization, J. Amer. Math. Soc., t. 1 (1988), pp. 867-918. 